\title{
Biocontrol Activity of Actinomycetes Strains against Fungal and Bacterial Pathogens of Solanum lycopersicum L. and Daucus carota L.: In Vitro and In Planta Antagonistic Activity ${ }^{\dagger}$
}

\author{
Rihab Djebaili ${ }^{1,2}, *\left(\mathbb{D}\right.$, Marika Pellegrini ${ }^{2}{ }^{-1}$, Matteo Bernardi ${ }^{2}$, Maria Smati $^{1}$, Mahmoud Kitouni ${ }^{1}$ \\ and Maddalena Del Gallo ${ }^{2}$ (i)
}

1 Laboratory of Microbiological Engineering and Applications, University of Brothers Mentouri, Constantine 1, Chaâbat Erssas Campus, Ain El Bey Road, Constantine 25000, Algeria; mariasmati87@gmail.com (M.S.); mahmoudkitouni@yahoo.fr (M.K.)

2 Department of Life, Health and Environmental Sciences, University of L'Aquila, Coppito 1, 67100 L'Aquila, Italy; marika.pellegrini@univaq.it (M.P.); matteo.bernardi1@graduate.univaq.it (M.B.); maddalena.delgallo@univaq.it (M.D.G.)

* Correspondence: djebaili.rihab@umc.edu.dz; Tel.: +39-086-243-3246

+ Presented at the 1st International Electronic Conference on Plant Science, 1-15 December 2020; Available online: https:/ / iecps2020.sciforum.net/.

check for

updates

Citation: Djebaili, R.; Pellegrini, M.; Bernardi, M.; Smati, M.; Kitouni, M.; Del Gallo, M. Biocontrol Activity of Actinomycetes Strains against Fungal and Bacterial Pathogens of Solanum lycopersicum L. and Daucus carota L. In Vitro and In Planta Antagonistic Activity. Biol. Life Sci. Forum 2021, 4, 27. https://doi.org/10.3390/ IECPS2020-08863

Academic Editor: Yoselin

Benitez-Alfonso

Published: 2 December 2020

Publisher's Note: MDPI stays neutral with regard to jurisdictional claims in published maps and institutional affiliations.

Copyright: (c) 2020 by the authors. Licensee MDPI, Basel, Switzerland. This article is an open access article distributed under the terms and conditions of the Creative Commons Attribution (CC BY) license (https:// creativecommons.org/licenses/by/ $4.0 /)$.

\begin{abstract}
Plants are affected by various biotic and abiotic stresses due to climate change. Tomato and carrots are important crops that are attacked by various pathogens. Fourteen plant-growth-promoting bacteria (PGPB) belonging to the genera Streptomyces sp. and Nocardiopsis sp. were selected for the biocontrol of several common fungal and bacterial pathogens. Antifungal activity was assessed against Fusarium oxysporum f. sp. radicis-lycopersici (FORL) and Rhizoctonia solani (RHS). Antibacterial activity was evaluated against Pseudomonas syringae (PS), Pseudomonas corrugata (PC), Pseudomonas syringae pv. actinidiae (PSA), and Pectobacterium carotovorum subsp. Carotovorum (PCC). In vitro antifungal and antibacterial antagonistic activities were evaluated by the dual culture method. Fungalbacterial interaction areas were analyzed by scanning electron microscopy (SEM). Cell-free culture filtrates (CFs) from strains showing good biocontrol potential were produced and investigated for their in vitro antifungal and antibacterial activity. The two most effective strains were also combined in consortium and utilized for In Planta pre-emergence biocontrol assays on both S. lycopersicum and D. carota. For each pathogenic strain, four experimental conditions were compared: CNT (no PGPB/no infection), PGPB (with PGPB/no infection), PGPB+INF (with PGPB/with infection), and INF (no PGPB/with infection). Streptomyces albidoflavus strain H12 and Nocardiopsis aegyptica strain H14 showed good in vitro antifungal (inhibition of $>50 \%$ ) and antibacterial (inhibition halo of $>10 \mathrm{~mm}$ ) activity. The SEM micrographs showed deterioration of fungal filaments and modification of hyphal structures. The CFs of both strains were also able to inhibit FORL and RHS in in vitro growth (minimum inhibitory concentration of $0.2-0.8 \%$ ). In planta biocontrol assessments showed that the consortium was effective in reducing the infection effects of both fungal and bacterial pathogens. Dual consortium allowed regular plant development compared to the control. These results confirm the usefulness of actinomycetes strains as a biocontrol agent and can therefore be an alternative to chemicals used in agriculture.
\end{abstract}

Keywords: PGPB; actinomycetes; biocontrol activity; fungal pathogens; pathogenic bacteria; SEM; culture filtrates; tomato; carrot

\section{Introduction}

Plants are affected by various biotic and abiotic stresses mostly due to climate change. Areas affected by temperature increase are more susceptible to pathogens attack [1]. The use of microorganisms in agriculture is a sustainable strategy to control phytopathogens. These 
bacteria can improve plant health and growth, providing long-term protection [2,3]. Several rhizospheric microorganisms act as biostimulants and show antagonistic properties against many pathogens [4]. Among them, actinomycetes have the ability to produce a wide range of secondary metabolites (e.g., antibiotics and extracellular enzymes) [5] which can inhibit the growth of several fungal and bacterial pathogens [6]. Moreover, biocontrol activity is obtained through the induction of systemic resistance [5]. The present study is aimed at evaluating the biocontrol capability of actinomycetes isolates against several fungal and bacterial pathogens of Solanum lycopersicum and Daucus carota. In vitro antifungal and antibacterial antagonistic activities were evaluated by the dual culture method. FungalPGPB interaction areas were also analyzed by scanning electron microscopy (SEM). From strains with good biocontrol potential, cell-free supernatants (CFSs) were produced and investigated for their in vitro antifungal and antibacterial activity. The most effective strains were also combined in consortium and utilized for the seed treatment for In Planta pre-emergence biocontrol assays on both S. lycopersicum and D. carota. For each pathogenic strain, four experimental conditions were compared: CNT (without PGPB/infection), PGPB (with PGPB/no infection), PGPB+INF (with PGPB/infection), and INF (with infection/no $\mathrm{PGPB})$. The induced protection was assessed by estimation of plant survival, morphobiochemical parameters, damage, and chlorophyll contents.

\section{Experimental Section}

In vitro antagonistic activity by diffusible and volatile compounds was carried out by the dual culture method on PDA medium using fourteen actinomycetes strains of the genus Streptomyces sp. and Nocardiopsis sp. The fungal pathogens tested were Fusarium oxysporum f. sp. radicis-lycopersici (FORL) and Rhizoctonia solani (RHS). The morphological deterioration of PGPB-fungus interaction areas was analyzed by SEM microscopy. The pathogenic bacteria tested were Pseudomonas syringae (PS), Pseudomonas corrugata (PC), Pseudomonas syringae pv. actinidiae (PSA), and Pectobacterium carotovorum subsp. carotovorum (PCC). The inhibition percentages of fungi were calculated after incubation until complete growth of the control plate [7]. Bacterial inhibition halos were assessed after $48 \mathrm{~h}$. Cell-free supernatants (CFSs) of Streptomyces albidoflavus $\mathrm{H} 12$ and Nocardiopsis aegyptica H14, which showed good in vitro biocontrol, were investigated for minimal inhibitory concentration (MIC) and for minimum bactericidal concentration (MBC) as described by CLSI guidelines [8], using sterile polystyrene microplate.

In planta antagonistic activity of $\mathrm{H} 12$ and $\mathrm{H} 14$ consortium, the most active strains in vitro, was assessed on S. lycopersicum and D. carota against the abovementioned pathogens in pre-emergence [7]. The experiment was organized as follows: (i) CNT (no PGPB/no infection), PGPB (with PGPB/no infection), PGPB+INF (with PGPB/with infection), and INF (no PGPB/with infection). Each experimental unit was realized in 25 pots with two seeds per pot under natural light conditions until the disease's symptoms showed up. The induced protection was assessed by estimation of plant survival, morpho-biochemical parameters, damage, and chlorophyll content. The plants were analyzed for the germination rates, morpho-physiological characteristics, damages, and total chlorophyll contents [7].

\section{Results}

Almost all 14 strains (64\%) showed good in vitro antagonistic activity by producing diffusible and volatile compounds against fungal pathogens (inhibition percentage up to $85 \%)$. Most of the tested strains (70\%) exhibited at least activity against one pathogenic bacterium (inhibition halo up to $25 \mathrm{~mm}$ ) (Table 1). 
Table 1. In vitro antagonism assay of actinomycetes strains against fungal and pathogenic bacteria $(n=3)$.

\begin{tabular}{ccccccccccccccc}
\hline Pathogenic Strains & D14 & G10 & G22 & G33 & H12 & H14 & J4 & J13 & J21 & J27 & K12 & K23 & S2 & T45 \\
\hline FORL & - & ++ & - & - & ++ & ++ & - & + & - & - & - & - & - & ++ \\
RHS & ++ & - & ++ & ++ & ++ & ++ & - & + & - & - & - & - & ++ & ++ \\
P. corrugata & - & ++ & - & ++ & ++ & ++ & - & ++ & & - & - & - & ++ & - \\
P. carotovorum & - & ++ & - & ++ & + & - & - & ++ & & + & - & - & - & - \\
\hline
\end{tabular}

+ , moderate inhibition;,++ high inhibition; -, no inhibition.

In Figure 1 SEM micrographs are shown which highlight the comparison of the structures of the fungal hyphae in the presence and absence of PGPB. Micrographs of FORL (Figure 1A) and RHS (Figure 1B) controls show normal and continuous fungal structures. The micrographs of FORL-PGPB (Figure 1C) and RHS-PGPB (Figure 1D) interaction areas show clear morphological deterioration of hyphae that appear sparse and discontinuous. S. albidoflavus $\mathrm{H} 12$ and N. aegyptica $\mathrm{H} 14$ also showed good antibacterial activity against Pseudomonas corrugata and Pectobacterium carotovorum (inhibition halos of $>10 \mathrm{~mm}$ ). CFSs of the combined strains were effective in inhibiting the growth of both FORL and RHS fungi (MBC up to $0.8 \%$ ) and most bacterial pathogen growth.
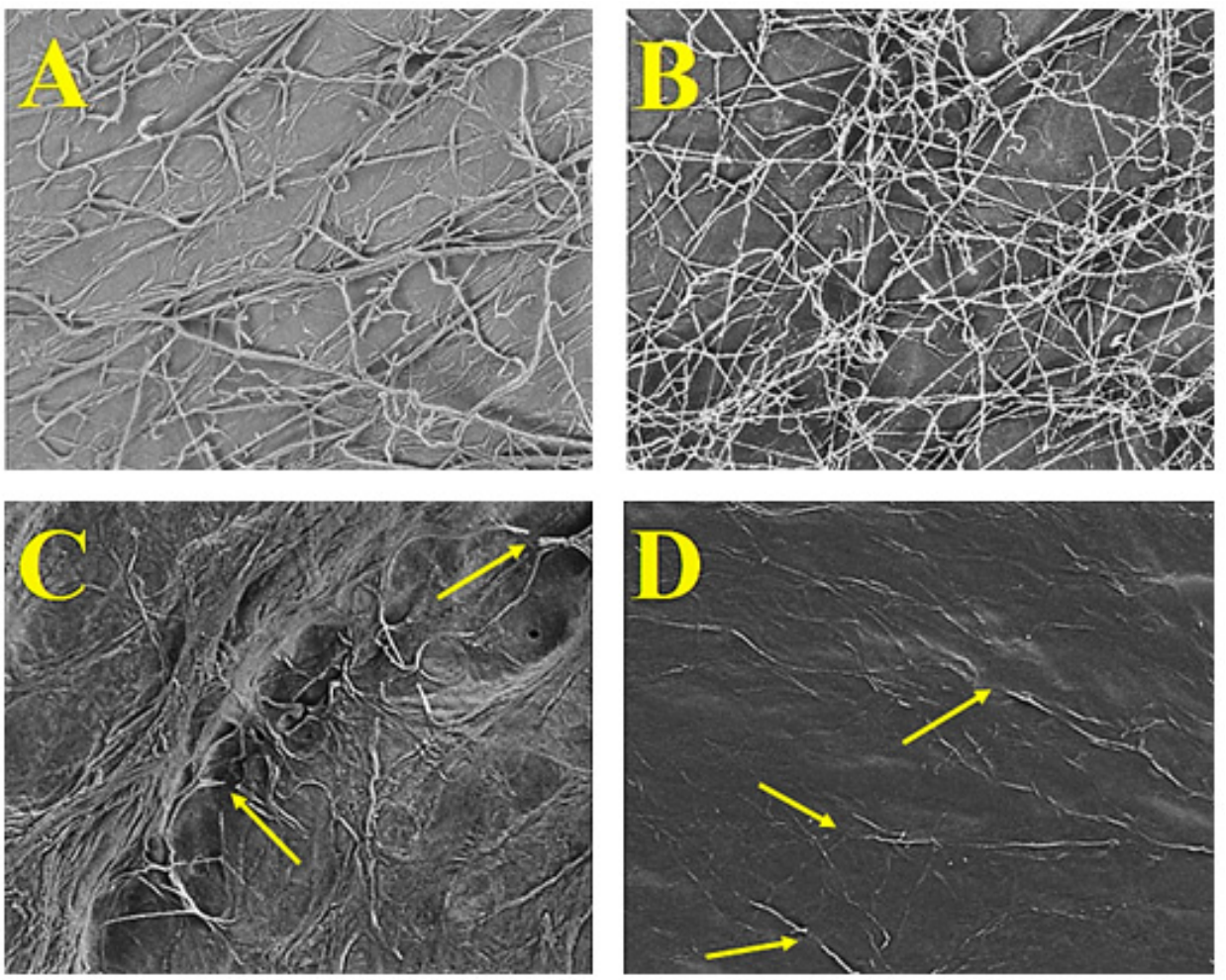

Figure 1. SEM micrographs of FORL and RHS hyphae. The panels show the fungal hyphal branching following normal growth in FORL and RHS control plates ((A,B), respectively). In the presence of the H12 and H14 consortium, the hyphal structures change in the interaction zones of both FORL and RHS in the presence of the PGPB $((\mathbf{C}, \mathbf{D})$, respectively).

Concerning the In Planta experiment, the inoculation with the consortium (PGPB) improved the development and growth of both tomato and carrot plants compared to the control (better germination rates, morpho-physiological characteristics, and chlorophyll content) (Figures 2 and 3). 

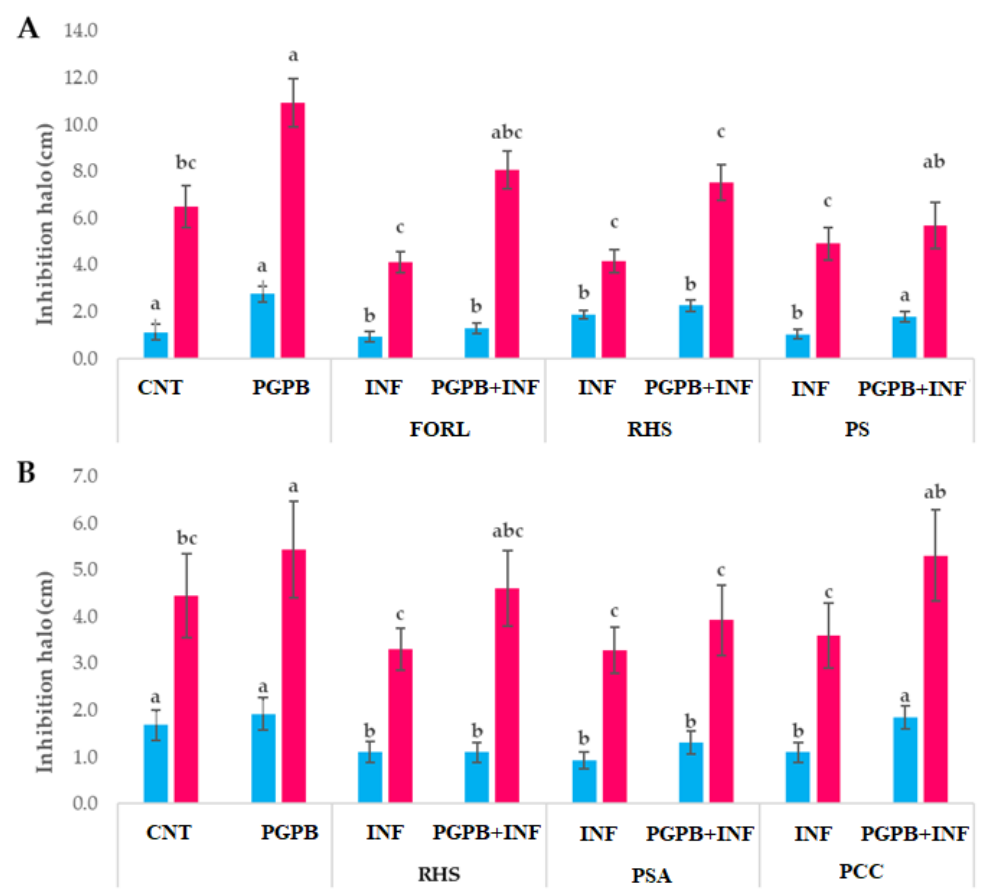

Figure 2. Shoot and root lengths (cm) of S. lycopersicum (A), and D. carota (B). Results followed by the same case letter are not significantly different according to Tukey's HSD post hoc test $(p>0.05)$. In the figure: PS, P. syringae; PSA, P. syringae pv. actinidiae; PCC P. carotovorum subsp. carotovorum.

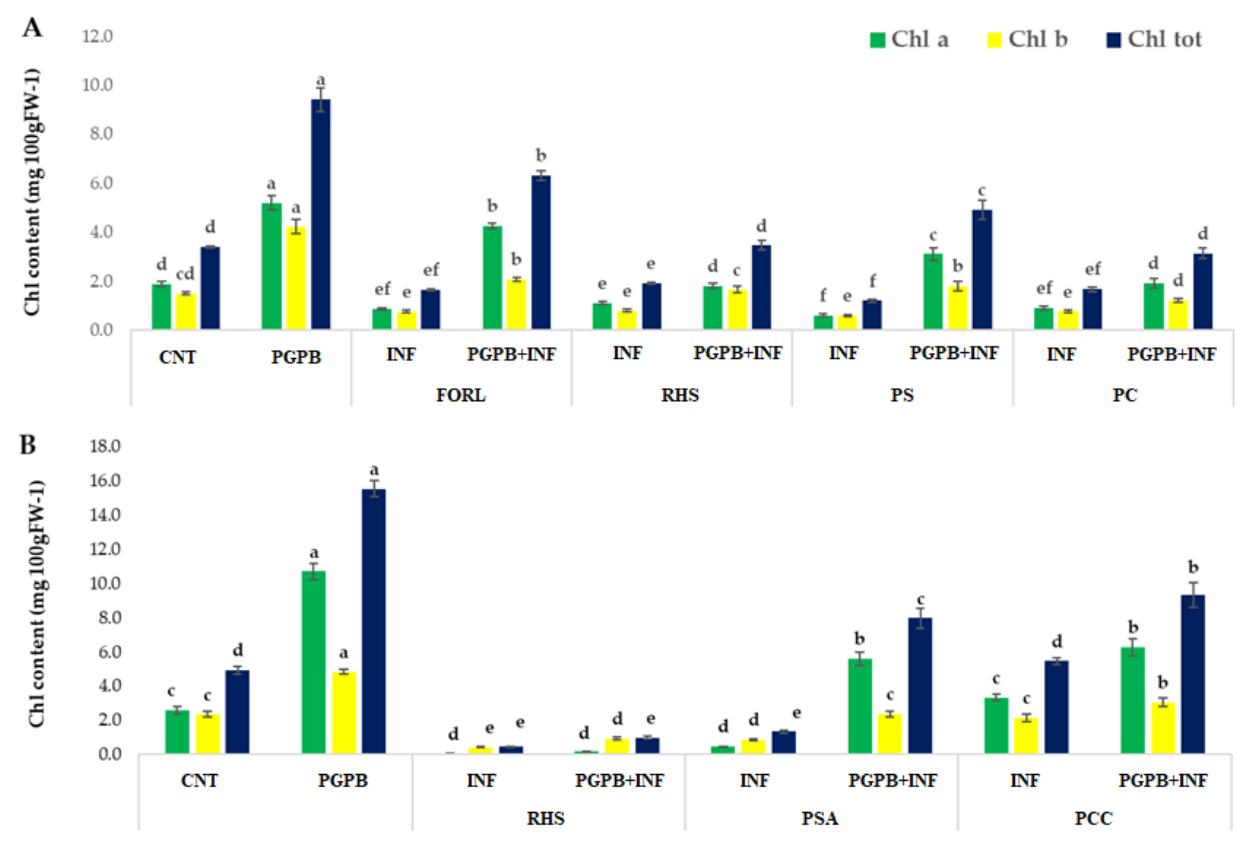

Figure 3. Chlorophyll b (chl a), chlorophyll b (chl b), and total chlorophyll (chl tot) content of tomato (A) and carrot (B) fresh leaves. Results followed by the same case letter are not significantly different according to Tukey's HSD post hoc test $(p>0.05)$. In the figure: PS, P. syringae; PC, P. corrugata; PSA, P. syringae pv. actinidiae; PCC P. carotovorum subsp. carotovorum.

Infection decreased germination rates, growth parameters, and total chlorophyll content, and caused leaf damage with extension up to $>20 \mathrm{~mm}$ in uninoculated plants (INF). Treatment with the consortium improved germination in infected plants (PGPB + INF) up to $15-54 \%$ for tomato and up to $30-100 \%$ for carrots. The presence of PGPB also alleviated infection symptoms: PGPB + INF plants showed less damage and better chlorophyll content than the control $(p<0.05)$. 


\section{Discussion}

The different abilities to promote plant growth by actinomycetes strains, such as nutrient solubilization, nitrogen fixation, and phytohormones production, act indirectly in the control of plant diseases [5]. The use of combined bacteria is a strategy for plant protection against pathogen attack [7]. Actinobacteria are well known for their ability to produce various bioactive compounds [9]. They are biological agents for their antagonistic activities and plant protection against several soil borne pathogens [10]. The biocontrol activity of actinomycetes is linked to antibiosis, lysis mechanisms, and host defenses induction [5]. The actinomycetes strains investigated in this study also have different plant growth-promoting traits [11]. These bacteria enhance plant physiological status and offer an additional advantage to their use as biological control agents for sustainable agriculture.

\section{Conclusions}

Nowadays, bacterial and fungal plant diseases are controlled almost exclusively by agrochemicals. These xenobiotic products entail serious consequences for human's and ecosystems' health. The use of biocontrol agents should be encouraged to counteract this problem. Our findings show that actinomycetes could be considered a valid biocontrol agent. Further experiments are needed to determine their effectiveness on other plants against other pathogens and under different cultivation conditions. However, these preliminary results underline that actinomycetes, mainly the genera Streptomyces and Nocardiopsis, may be biological alternatives for the management of plant disease.

Supplementary Materials: The poster presentation is available online at https:/ /www.mdpi.com/ article/10.3390/IECPS2020-08863/s1.

Author Contributions: Conceptualization, R.D., M.P. and M.D.G.; methodology, M.S. and M.K.; formal analysis, R.D. and M.B.; investigation, R.D. and M.P.; resources, M.K. and M.D.G.; data curation, R.D. and M.P.; writing—original draft preparation, R.D. and M.P.; writing—review and editing, M.D.G. All authors have read and agreed to the published version of the manuscript.

Funding: This research received no external funding.

Institutional Review Board Statement: Not applicable.

Informed Consent Statement: Not applicable.

Acknowledgments: We wish to thank Lorenzo Arrizza for the support in the Scanning Electron Microscope analysis.

Conflicts of Interest: The authors declare no conflict of interest.

\section{References}

1. Nawaz, R.; Abbasi, N.A.; Hafiz, I.A.; Khalid, A. Increasing level of abiotic and biotic stress on Kinnow fruit quality at different ecological zones in climate change scenario. Environ. Exp. Bot. 2020, 171, 103936. [CrossRef]

2. Fernando, W.G.D.; Ramarathnam, R.; Krishnamoorthy, A.S.; Savchuk, S.C. Identification and use of potential bacterial organic antifungal volatiles in biocontrol. Soil Biol. Biochem. 2005, 37, 955-964. [CrossRef]

3. Orio, A.G.A.; Brücher, E.; Ducasse, D.A. A strain of Bacillus subtilis subsp. subtilis shows a specific antagonistic activity against the soil-borne pathogen of onion Setophoma terrestris. Eur. J. Plant Pathol. 2016, 144, 217-223. [CrossRef]

4. Chauhan, A.K.; Maheshwari, D.K.; Kim, K.; Bajpai, V.K. Termitarium-inhabiting Bacillus endophyticus TSH42 and Bacillus cereus TSH77 colonizing Curcuma longa L.: Isolation, characterization, and evaluation of their biocontrol and plant-growth-promoting activities. Can. J. Microbiol. 2016, 62, 880-892. [CrossRef] [PubMed]

5. Hata, E.M.; Sijam, K.; Ahmad, Z.A.M.; Yusof, M.T.; Azman, N.A. In vitro Antimicrobial Assay of Actinomycetes in Rice Against Xanthomonas oryzae pv. oryzicola and as Potential Plant Growth Promoter. Braz. Arch. Biol. Technol. 2015, 58, 821-832. [CrossRef]

6. Hasegawa, S.; Meguro, A.; Shimizu, M.; Nishimura, T.; Kunoh, H. Endophytic Actinomycetes and Their Interactions with Host Plants. Actinomycetologica 2006, 20, 72-81. [CrossRef]

7. Pellegrini, M.; Ercole, C.; Di Zio, C.; Matteucci, F.; Pace, L.; Del Gallo, M. In vitro and in planta antagonistic effects of plant growth-promoting rhizobacteria consortium against soilborne plant pathogens of Solanum tuberosum and Solanum lycopersicum. FEMS Microbiol. Lett. 2020, 367, 99. [CrossRef] [PubMed]

8. Clinical and Laboratory Standards Institute. Performance Standards for Antimicrobial Susceptibility Testing; Twenty-First Informational Supplement; CLSI: Wayne, PA, USA, 2011; Volume 31, ISBN 1562387421. 
9. Ansari, W.A.; Krishna, R.; Zeyad, M.T.; Singh, S.; Yadav, A. Endophytic Actinomycetes-Mediated Modulation of Defense and Systemic Resistance Confers Host Plant Fitness Under Biotic Stress Conditions. In Microbial Versatility in Varied Environments; Springer: Singapore, 2020; pp. 167-180.

10. Vurukonda, S.S.K.P.; Giovanardi, D.; Stefani, E. Plant Growth Promoting and Biocontrol Activity of Streptomyces spp. as Endophytes. Int. J. Mol. Sci. 2018, 19, 952. [CrossRef] [PubMed]

11. Djebaili, R.; Pellegrini, M.; Smati, M.; Del Gallo, M.; Kitouni, M. Actinomycete Strains Isolated from Saline Soils: Plant-GrowthPromoting Traits and Inoculation Effects on Solanum lycopersicum. Sustainability 2020, 12, 4617. [CrossRef] 